ciency but a low serum folate as suggesting a negative folate balance which in time may lead to true deficiency.

Maintenance of a normal serum level of folate depends in part on a constant supply of folate from the diet. During the course of normal absorption it is converted to methyltetrahydrofolate in the gut wall, ${ }^{13}$ and this form tends to persist in plasma because its clearance is relatively slow. ${ }^{14}$ Illhealth with loss of appetite may reduce dietary intake, curtail the supply of folate from the diet, and so lead to a decline in its concentration in plasma. This is likely to precede any significant depletion of hepatic and other folate stores in the body. ${ }^{15}$

R. Gräsbeck and colleagues ${ }^{16}$ first drew attention to an unusually raised urinary excretion of formiminoglutamic acid in five patients with congestive cardiac failure. Excretion returned to normal when the patients were treated with folate. An increased urinary excretion of urocanic acid, the precursor of formiminoglutamic acid, was noted by J. J. Daly and D. P. Rose. ${ }^{17}$ Two other folate studies in cardiac failure have appeared in recent years. R. D. Hyde and C. A. E. H. Loehry ${ }^{18}$ reported on 24 patients. The serum level of folate ranged from $2 \cdot 1$ to $9 \cdot 0 \mathrm{ng}$. per $\mathrm{ml}$. and in all was within the normal range. The blood film showed iron deficiency in three patients but was normal in the remainder. However, impaired absorption of folate was noted in seven patients, and in these the absorption improved when the heart failure was relieved. Two of their patients also had steatorrhoea. By contrast J. I. Brody and colleagues ${ }^{10}$ noted reduced levels of serum folate in 9 out of 12 patients in cardiac failure; these coupled with minor changes in blood and marrow films led them to interpret their data as indicating significant folate deficiency. Too much emphasis should not be put on serum folate values alone and it is not clear to what extent the serum folate values reported by Brody and colleagues differed from those of hospital patients in general. Red-cell folate values are the important ones to use as the criterion of deficiency in such studies.

Nevertheless it seems clear that minor variations in folate metabolism are common in patients with cardiac failure. They may arise from an impaired dietary intake of folate just before the development of severe failure, but absorption of folate may be impaired in some cases. These biochemical aberrations are not in themselves an indication for folate therapy. They are likely to be reversed by treating the cardiac failure and restoring a normal appetite and hence normal food intake. Anaemia, if present, is usually due to iron deficiency. It would be unwise to attribute megaloblastic anaemia to congestive cardiac failure alone without careful study of intestinal function, nutritional status, effects of medication, and all the other factors that can contribute to megaloblastic haemopoiesis.

\section{Atrial Function in Man}

The atrium has three main functions apart from its role as a pipeline in directing blood to the ventricles. Firstly, as a "reservoir" it ensures that blood flowing relatively slowly in the venous system is available in adequate quantities to fill the ventricle during the rapid filling phase at the start of diastole. Investigations in dogs have shown that at rest this function is unimpaired until half of the left atrial volume is lost. ${ }^{2}$ Though great caution must be used in applying these results to man, it seems unlikely that volume changes due to surgery, blood clot, or tumours will cause symptoms.

Secondly, the atria in systole have a role in closing the atrioventricular valves, but this also seems to be unimportant clinically. There is no doubt that mitral and tricuspid valves often close during the short period of pressure reversal between atrial and ventricular contractions, but this is not an essential mechanism for satisfactory closure of the mitral valve in man. ${ }^{3}$

Thirdly, and of greater significance, is the atrial transport mechanism. A correctly timed atrial contraction augments ventricular filling and increases ventricular end-diastolic pressure, thus maintaining subsequent ventricular stroke volume and systemic blood pressure. These effects are particularly important when cardiac function is poor, ${ }^{4}$ when ventricular filling is obstructed, or when diastolic filling time is reduced by tachycardia. Alterations in the timing of atrial systole occur during atrioventricular dissociation or complete heart block and may result in temporary hypotension, particularly after cardiac infarction. ${ }^{5}$ Pressure changes such as these do not persist long enough for the haemodynamic results to be modified by compensatory mechanisms, and changes may be severe enough for patients to lose consciousness.

There are only a few investigations in man into the effects of atrial transport on cardiac output. P. Samet and colleagues $^{6}$ found that the mean increase in output for a group of patients when atrial systole was correctly timed was $10 \%$, but there were considerable differences between patients and some obtained no benefit at all. A. Benchimol and $M$. S. Liggett ${ }^{7}$ studied normal persons and patients with coronary artery disease and concluded that atrial transport was of little importance when cardiac function was good. Similar studies have shown that cardiac output during exercise is greater when atrioventricular timing is normal than when it is random or absent.

Studies of ventricular function before and after electrical reversion from atrial fibrillation to sinus rhythm have shed further light on the importance of atrial transport. L. Resnekov ${ }^{8}$ showed that patients had smaller stroke volumes and poorer exercise tolerance during atrial fibrillation than sinus rhythm, and that the improvement was not due only to differences in ventricular rate. The patients received little benefit at rest.

Information about the long-term effects of atrial deprivation can be obtained from patients with atrial standstill. One such patient in whom absence of atrial contraction was recorded for eight years has recently been reported on by W. J. Messinger and A. M. Mirkinson.' This 47-year-old man had many electrocardiographs done during admissions to hospital for hypotension and obscure chest pain, but atrial activity was never detected in any lead, including oesophageal recordings, and no atrial activity could be seen

1 Grant, C., Bunnell, I. L., and Greene, D. G., American fournal of Medicine, 1964, 37. 36.

Trusler, G. A., Bull, R. C., Hoeksema, T., and Mustard, W. T., fournal of Thoracic and Cardiovascular Surgery, 1963, 46, 109.

3 Braunwald, E., Rockoff, S. D., Oldham, H. N., Jr., and Ross, J., Jr., Circulation, 1966, 33, 404.

Burchell, H. B., Lancet, 1964, 1, 775.

5 Sowton, E., in Acute Myocardial Infarction, p. 156, ed. D. G. Julian and $M$. F. Oliver. Edinburgh, Livingstone. 1968 .

6 Samet, P., Bernstein, W. H., Nathan, D. A., and López, A., American fournal of Cardiology, 1965, 16, 1 .

7 Benchimol, A., and Liggett, M. S., Circulation, 1966, 33, 933.

\& Resnekov, L.. British Heart fournal, 1967, 29, 700.

Messinger, W. J., and Mirkinson, A. M., Archives of Internal Medicine, 1969, 124, 211 .

in Chavez, I., Brumlik, J., and Sodi-Pallares, D. S., Archivos del Instituto de Cardiologia de México, 1946, 16, 159. 
during fluoroscopy with an intracardiac lead against the right atrial border. Although there can be no doubt that his cardiac function was poor, the patient managed normal activities, including a full-time job. Other similar patients have been described, ${ }^{10}$ and it is apparent that absence of atrial contraction is compatible with moderate activity provided cardiac function is not grossly impaired.

Clinically, the loss of atrial transport makes little difference to most patients at rest unless they have had a recent cardiac infarction or are in heart failure. Dependence of cardiac performance on atrial transport is most noticeable in patients whose output is already low, and atrial transport may be important after cardiac surgery for this reason. The $10-25 \%$ reduction in cardiac output during effort may cause rapid deterioration in patients with valve disease, cardiomyopathy, or intracardiac shunts even if the ventricular rate is well controlled. Although loss of atrial transport is a small matter to a normal heart it may be the final insult to an ill patient, and it is under these conditions that vigorous attempts should be made to restore normal atrial performance.

\section{Oral Contraceptives and Gestational Diabetes}

Since V. Wynn and J. W. H. Doar ${ }^{1}$ drew attention to the possible effects of oral contraceptives on carbohydrate metabolism, physicians in Britain have been chary of prescribing them for women with overt or subclinical diabetes. Many women develop some degree of carbohydrate intolerance during pregnancy and then revert to normal in the puerperium. They have been called "gestational diabetics." Some ask for contraceptive advice, and it is difficult to decide whether oral contraceptives might safely be prescribed for them. A recent study by $P$. Beck and S. A. Wells ${ }^{2}$ in the United States carries the matter a little forward. What they set out to do was to see what effect oral contraceptives would have on carbohydrate metabolism during the puerperium in women who showed signs of subclinical diabetes.

The matter is not easy to examine scientifically. Firstly, one needs to examine how long an oral contraceptive has to be used before any effect can be observed, and secondly one needs to define the nature of the oral contraceptive being tested. Are the effects due to the oestrogen or to the progestogen? P. Beck and S. A. Wells ${ }^{2}$ studied the most commonly used oestrogen, mestranol, and two progestogens, ethynodiol diacetate and chlormadinone. Their patients were examined after 2,10 , and 22 weeks' treatment. Though some previous work had suggested that different progestogens had different effects, ${ }^{3}$ they did not find any differences between these two and were inclined to ascribe most of the effects they found to the oestrogen.

Beck and Wells found their group of gestational diabetics by selecting them from 26 women on whom oral glucosetolerance tests were done in the third trimester of pregnancy.

\footnotetext{
1 Wynn, V., and Doar, J. W. H., Lancet, 1966, 2, 715.

2 Beck, P., and Wells, S. A., Fournal of Clinical Endocrinology and Metabolism, 1969, 29, 807.

3 Spellacv, W. N. In Kipnis, D. M.. Salhanick, H. A., and van de Wiele, R. M., Metabolic Effects of Gonadal Hormones and Contracertive R. M., Metabolic Effects of Gonadal Horn
Steroids. New York, Plenum Press. 1969.

4 Clinch, J., Turnbull, A. C., and Khosla, T., Lancet, 1969, 1, 857.
}

Twelve of them showed an abnormal blood-sugar response to a glucose load but had reverted to normal when retested in the fifth week of the puerperium. The remaining 14 patients, who were normal on both occasions, formed a convenient control group. All the patients were then tested a third time after two weeks' treatment with mestranol or mestranol plus ethynodiol diacetate. After only two weeks on steroids the glucose tolerance had changed for the worse in the gestational diabetics. Two of them now had frankly abnormal glucose tolerance and a further four showed some deterioration in their capacity to metabolize a glucose load. J. Clinch, and colleagues ${ }^{4}$ have found that the effect of oral contraceptives on glucose tolerance in normal women is dependent on dose; indeed very small doses improved the carbohydrate tolerance. But it is not surprising that Beck and Wells should have found such a definite deterioration, for they were using larger doses and testing patients who had already shown some evidence of deficiency in their capacity to deal with a glucose load during pregnancy.

Because they had tested their patients during pregnancy and also did prednisolone provocation tests in the puerperium before starting the oral contraceptives, Beck and Wells could make interesting comparisons between the effects on carbohydrate metabolism of pregnancy, of prednisolone, and of oral contraceptives both in normal women and in gestational diabetics. In the latter group pregnancy and glucocorticoids caused a fall in the rate of insulin secretion, so that the increased insulin response in the first hour after an oral glucose load was less than in normal women. By contrast oral contraceptives produced no fall in the initial response of insulin secretion either in the gestational diabetics or in the normal women. From experiments on monkeys Beck and Wells are inclined to believe that mestranol causes a decreased sensitivity to the hypoglycaemic effect of normal insulin.

In the light of all this, should oral contraceptives be prescribed for women in whom gestational diabetes has been discovered? A firm answer seems impossible to give at present. The findings so far suggest that for these patients doctors should exercise special care in the prescribing of them and be the more reluctant to do so when other forms of contraceptives are acceptable. If oral contraceptives are to be prescribed it might be wise to avoid the use of mixtures containing oestrogen and perhaps to rely on small doses of pure progestational compounds such as clomadinone acetate.

\section{February S.R.M.}

An emergency meeting of the Council on 16 January decided to call a Special Representative Meeting on 12 February to settle the Association's policy on the General Medical Council's proposed annual retention fee and on the number of the profession's elected representatives on the G.M.C. The notice convening the Meeting and a report by the Council are printed in the Supplement.

We regret that again owing to a plant breakdown some copies of the 17 January issue of the B.M.f. were delivered late. 